\title{
Author Correction: Taking atom interferometric quantum sensors from the laboratory to real-world applications
}

Kai Bongs (D), Michael Holynski(D, Jamie Vovrosh (D), Philippe Bouyer(D), Gabriel Condon, Ernst Rasel(D), Christian Schubert, Wolfgang P. Schleich (D) and Albert Roura (D)

Correction to: Nature Reviews Physics https://doi.org/10.1038/s42254-019-0117-4, published online 28 October 2019.

In the original version of this article, the second affiliation of Albert Roura was missing. The second affiliation, German Aerospace Center (DLR), Cologne, Germany, has now been added. This error has now been corrected in the HTML and PDF versions of the article.

https://doi.org/10.1038/s42254-021-00396-1 I Published online 3 November 2021

๑) Springer Nature Limited 2021 